\title{
SYNTHESIS AND EVALUATION OF HEPATOPROTECTIVE ACTIVITY OF SOME NEW MANNICH BASES BEARING BENZTRIAZOLE MOIETY
}

\author{
${ }^{1}$ AIYALU RAJASEKARAN* AND ${ }^{2}$ MUTHUSAMY PERIYASAMY , ${ }^{1} \mathrm{KMCH}$ COLLEGE OF PHARMACY, \\ COIMBATORE INDIA., ${ }^{2}$ ERODE COLLEGE OF PHARMACY, ERODE, INDIA
}

Dr. Aiyalu Rajasekaran.M.Pharm, Ph.D., Professor and Principal, KMCH College of Pharmacy, Kovai Estate, Kalapatti Road, Coimbatore - 641048

(Received: December 29, 2009 - Accepted: July 22, 2010)

\begin{abstract}
A series of benztriazoles bearing Mannich bases (2 -10) were synthesized from benztriazole by aminomethylation with formaldehyde and various substituted secondary amines. Titled compounds were synthesized by Mannich reaction and they were characterized by IR and ${ }^{1} \mathrm{HNMR}$ spectroscopy. All these Mannich bases were screened for hepatoprotective activity on carbon tetrachloride induced liver damage in rats. Only compounds $4(250 \mathrm{mg} / \mathrm{kg}) \mathrm{protected} \mathrm{significantly} \mathrm{the}$ animals from carbon tetrachloride induced hepatotoxicity. Compound $4 \mathrm{~N}$-morpholinyl methyl benztriazole exhibited significant activity comparable to that of standard drug silymarin.
\end{abstract}

Keywords: Mannich bases, benztriazoles, hepatoprotective, Serum Glutamate Oxaloacetate Transaminase, Serum Glutamate Pyruvate Transaminase, Alkaline Phosphatase .

\section{INTRODUCTION}

Liver is playing important role in metabolism, secretion and storage. Any injury to liver can result in many disorders ranging from elevation of liver enzymes to liver failure. Commonly, liver injuries are caused by toxic chemicals like carbon tetrachloride $\left(\mathrm{CCl}_{4}\right)$, aflatoxin and therapeutic agents like antibiotic, anti-tubercular drugs as well as alcohol, microbial agents [1]. Administration of $\mathrm{CCl}_{4}$ is biotransformed by cytochrome P-450 system to produce the trichloromethyl free radical which causes lipid peroxidation and membrane damage. Also, $\mathrm{CCl}_{4}$ increases the serum level of marker enzymes, serum glutamate pyruvate transaminase (SGPT), serum glutamate oxaloacetate transaminase (SGOT) and alkaline phosphatase (ALP) indicating the induction of hepatotoxicity [2]. Silymarin has proved to possess multiple actions as a hepatoprotective against various hepatotoxic substances in its preclinical studies. The antioxidant property and cell-regenerating functions as a result of increased protein synthesis are considered as most important. It can prevent the absorption of toxins into the hepatocytes by occupying the binding sites as well as inhibiting many transport proteins at the membrane. These actions along with antiperoxidative property make silymarin a suitable candidate for the treatment of iatrogenic and toxic liver diseases [3].

Mannich reaction is an important tool for synthesis of novel compounds. Mannich bases are physiologically reactive because of the basic function rendering the molecule soluble in aqueous solvents when it is transformed into aminium salt. Mannich bases have been reported to possess antibacterial [46], antitubercular [7-9], antimalarial [10-11], anti-HIV [12] and analgesic and antinflammatory [13-14] activities. 1,2,4-triazole nucleus has been incorporated into wide variety of therapeutically interesting molecules to transform them into better drugs. Various 1,2,4-triazole derivatives have been reported to possess anticancer [15], antioxidant [16], antibacterial [17], antifungal [18], analgesic [19], anti-inflammatory [20] and anti-emetic activity [21]. Mannich bases of 1,2,4-triazole were reported for anticancer [22] activity, protozocidal and antibacterial activity [23]. Some of the modern-day drugs with triazole nucleus are the antiviral agent Ribavirin, antimigrane agent Rizatriptan, anxiolytic agent Alprazolam, antifungal agents Flucanozole and Itraconazole. Amino azoles are reported for potential hepatoprotectant activity [24]. There is no report on the synthesis and hepatoprotective evaluation of Mannich bases of 1,2,4-benztriazoles. Hence it was contemplated to synthesize some congeners of benztriazole containing Mannich bases with a view to explore their potency as hepatoprotective agents.

\section{MATERIALS AND METHODS}

All melting points were taken by open capillary tubes and were uncorrected. Thin layer chromatography was performed on precoated Silica Gel $60 \mathrm{~F}_{254}$ plates from E.Merck using methanol and benzene as mobile phase (50:50) and visualized by exposure to iodine vapors. IR spectra recorded on a Perkin Elmer IR spectrophotometer, using $\mathrm{KBr}$ pellets. NMR spectra were recorded on a Bruker DRX 300 (300MHZ) NMR spectrophotometer in $\mathrm{CDCl}_{3}$ using TMS as internal standard. Elemental analysis was performed on Heraceus Carlo Erba 1108 and the analysis indicated by the symbols of the elements was within \pm $0.4 \%$ of theoretical values.

General procedure for the synthesis of Mannich bases of benztriazole Benztriazole $(0.01 \mathrm{~mol})$ was dissolved in methanol $(50 \mathrm{~mL})$ in a $250 \mathrm{~mL}$ beaker under ice cold condition. Various secondary amines $(0.01 \mathrm{~mol})$ were added in small quantities with constant stirring under ice cold condition. A measured quantity of formaldehyde $(0.01 \mathrm{~mol})$ was added, slowly with constant stirring for $4 \mathrm{hrs}$. The contents of the beaker were kept over night in the freezer. Crystallized product was filtered and dried. The product was recrystallized using methanol. The reaction sequence is outlined in Scheme 1. The yield, melting point and Rf value were reported in Table 1 .

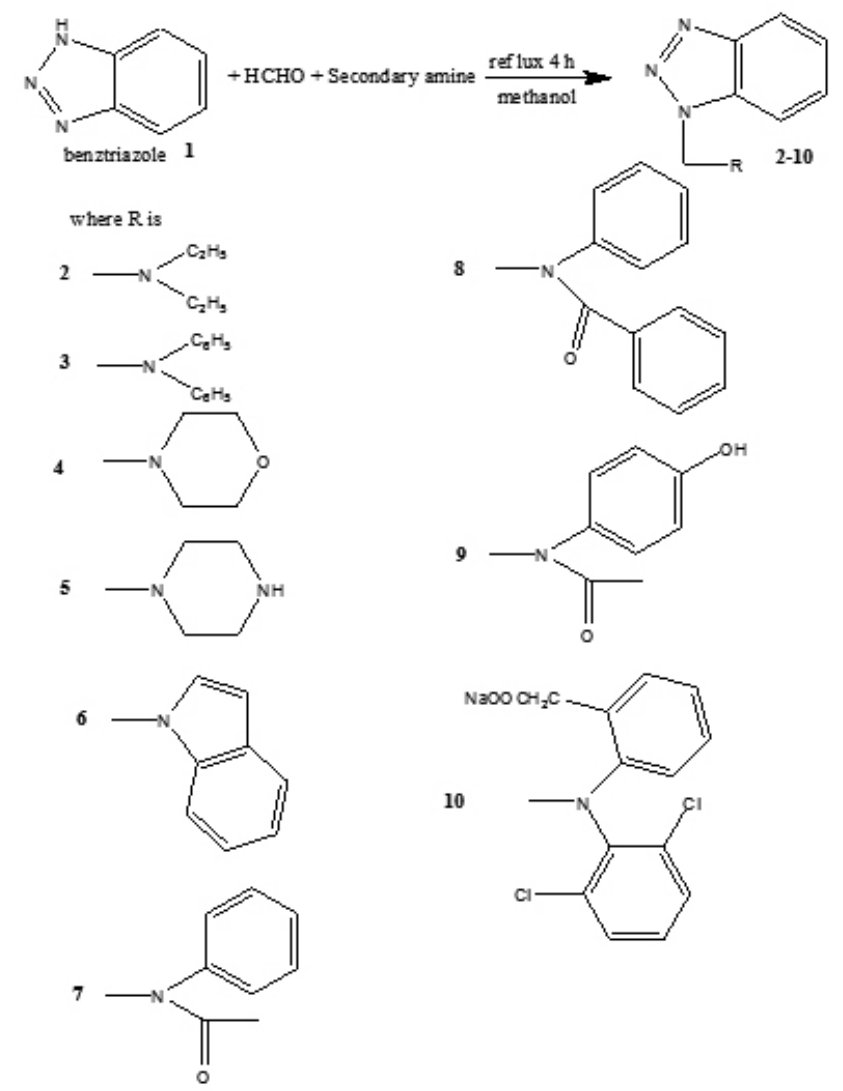

Sheme 1: Synthetic protocol of benztriazole Mannich bases. 
Table 1: Physical and analytical data of newly synthesized compounds.

\begin{tabular}{|c|c|c|c|c|c|c|}
\hline Compound & $\mathrm{R}$ & M.P. $\left({ }^{\circ} \mathrm{C}\right)$ & Yield (\%) & Rf value* & Molecular formula & Mol Weight \\
\hline 2 & & $100-102$ & 48 & 0.2 & $\mathrm{C}_{11} \mathrm{H}_{16} \mathrm{~N}_{4}$ & 204.27 \\
\hline 3 & & ------ & 62 & 0.67 & $\mathrm{C}_{19} \mathrm{H}_{16} \mathrm{~N}_{4}$ & 300.35 \\
\hline 4 & & $80-82$ & 54 & 0.4 & $\mathrm{C}_{11} \mathrm{H}_{14} \mathrm{~N}_{4} \mathrm{O}$ & 218.25 \\
\hline 5 & & $172-174$ & 70 & 0.91 & $\mathrm{C}_{11} \mathrm{H}_{15} \mathrm{~N}_{5}$ & 217.27 \\
\hline 6 & & $110-112$ & 60 & 0.70 & $\mathrm{C}_{15} \mathrm{H}_{12} \mathrm{~N}_{4}$ & 248.28 \\
\hline 7 & & $82-84$ & 59 & 0.78 & $\mathrm{C}_{15} \mathrm{H}_{14} \mathrm{~N}_{4} \mathrm{O}$ & 266.29 \\
\hline 8 & & $165-168$ & 64 & 0.73 & $\mathrm{C}_{20} \mathrm{H}_{16} \mathrm{~N}_{4} \mathrm{O}$ & 328.36 \\
\hline 9 & & $120-122$ & 72 & 0.34 & $\mathrm{C}_{15} \mathrm{H}_{14} \mathrm{~N}_{4} \mathrm{O}_{2}$ & 282.29 \\
\hline 10 & & $220-222$ & 68 & 0.45 & $\mathrm{C}_{21} \mathrm{H}_{15} \mathrm{Cl}_{2} \mathrm{~N}_{4} \mathrm{NaO}_{2}$ & 449.26 \\
\hline
\end{tabular}

*chloroform and benzene as mobile phase, spot detection-Iodine vapour 
Infrared spectra of all compounds showed strong absorptions at 2860 and $2846 \mathrm{~cm}^{-1}$ for the $\mathrm{CH}_{2}$ group (Mannich methylene). ${ }^{1} \mathrm{HNMR}$ spectra all compounds showed a singlet at $d 4.2 \mathrm{ppm}$ for methylene protons of $-\mathrm{NCH}_{2} \mathrm{~N}-$ group.

Compound 2

IR $(\mathrm{KBr}) \mathrm{n} / \mathrm{cm}^{-1}: 3099$ (Ar-H), 2975(C-H), $1640(\mathrm{C}-\mathrm{N}), 1600(\mathrm{C}=\mathrm{C})$. ${ }^{1} \mathrm{HNMR}\left(d, \mathrm{CDCl}_{3}\right) ; 2.9$ (t, $\left.6 \mathrm{H},\left(\mathrm{CH}_{2}-\mathrm{CH}_{3}\right)_{2}\right), 3.2\left(\mathrm{q}, 4 \mathrm{H},\left(\mathrm{CH}_{2}-\mathrm{CH}_{3}\right)_{2}\right), 7.4-7.9$ (m, 4H, Ar-H).

Compound 3

IR (KBr) n/ cm cm $^{-1} 078(\mathrm{Ar}-\mathrm{H}), 2998(\mathrm{C}-\mathrm{H}), 1656(\mathrm{C}-\mathrm{N}), 1500(\mathrm{C}=\mathrm{C})$.

${ }^{1} \mathrm{HNMR}\left(\mathrm{d}, \mathrm{CDCl}_{3}\right) ; 6.7-7.9$ (m, 14H, Ar-H).

Compound 4

IR $(\mathrm{KBr}) \mathrm{n} / \mathrm{cm}^{-1}: 3102$ (Ar-H), 2984(C-H), $1644(\mathrm{C}-\mathrm{N}), 1540(\mathrm{C}=\mathrm{C})$. ${ }^{1} \mathrm{HNMR}\left(d, \mathrm{CDCl}_{3}\right) ; 2.5\left(\mathrm{t}, 4 \mathrm{H},\left(-\mathrm{N}-\mathrm{CH}_{2}-\mathrm{CH}_{2}-\mathrm{O}-\right), 3.7\left(\mathrm{t}, 4 \mathrm{H},\left(-\mathrm{N}-\mathrm{CH}_{2}-\right.\right.\right.$ $\left.\mathrm{CH}_{2}-\mathrm{O}-\right)$, 7.4-7.9 (m, 4H, Ar-H).

Compound 5

IR (KBr) n/ cm ${ }^{-1}: 3325$ (NH), 3098 (Ar-H), 2974(C-H), 1654 (C-N), 1500

$(\mathrm{C}=\mathrm{C}) .{ }^{1} \mathrm{HNMR}\left(d, \mathrm{CDCl}_{3}\right) ; 2.4\left(\mathrm{t}, 4 \mathrm{H},\left(-\mathrm{N}-\mathrm{CH}_{2}-\mathrm{CH}_{2}-\mathrm{N}-\right), 2.7\left(\mathrm{t}, 4 \mathrm{H},\left(-\mathrm{N}-\mathrm{CH}_{2}-\right.\right.\right.$ $\left.\mathrm{CH}_{2}-\mathrm{N}-\right)$, multiplet at d 2.46-3.45 ppm for piperazine proton, 7.4-7.9 (m, $4 \mathrm{H}^{2}$, $\mathrm{Ar}-\mathrm{H})$.

Compound 6

IR $(\mathrm{KBr}) \mathrm{n} / \mathrm{cm}^{-1}: 3094$ (Ar-H), 2978(C-H), $1650(\mathrm{C}-\mathrm{N}), 1570(\mathrm{C}=\mathrm{C})$.

${ }^{1} \mathrm{HNMR}\left(d, \mathrm{CDCl}_{3}\right)$ 6.4-7.6 (m, 10H, Ar-H).

Compound 7

IR (KBr) n/ cm ${ }^{-1}: 3089$ (Ar-H), 2987(C-H), $1694(\mathrm{C}=\mathrm{O}), 1642(\mathrm{C}-\mathrm{N})$, $1570(\mathrm{C}=\mathrm{C}) .{ }^{1} \mathrm{HNMR}\left(d, \mathrm{CDCl}_{3}\right) 2.0\left(\mathrm{~s}, 3 \mathrm{H}, \mathrm{CH}_{3}\right), 6.4-7.6(\mathrm{~m}, 9 \mathrm{H}, \mathrm{Ar}-\mathrm{H})$.

Compound 8

IR (KBr) n/ cm ${ }^{-1}: 3078$ (Ar-H), 2983(C-H), $1680(\mathrm{C}=\mathrm{O}), 1624(\mathrm{C}-\mathrm{N})$,

$1590(\mathrm{C}=\mathrm{C}) .{ }^{1} \mathrm{HNMR}\left(d, \mathrm{CDCl}_{3}\right)$ 6.4-7.9 (m, 14H, Ar-H).

Compound 9

IR $(\mathrm{KBr}) \mathrm{n} / \mathrm{cm}^{-1}: 3556(\mathrm{OH}), 3088(\mathrm{Ar}-\mathrm{H}), 2992(\mathrm{C}-\mathrm{H}), 1676(\mathrm{C}=\mathrm{O}), 1641$

$(\mathrm{C}-\mathrm{N}), 1590(\mathrm{C}=\mathrm{C}) .{ }^{1} \mathrm{HNMR}\left(d, \mathrm{CDCl}_{3}\right) 2.0\left(\mathrm{~s}, 3 \mathrm{H}, \mathrm{CH}_{3}\right), 6.4-7.9(\mathrm{~m}, 8 \mathrm{H}, \mathrm{Ar}-$ $\mathrm{H})$.

Compound $\mathbf{1 0}$

IR $(\mathrm{KBr}) \mathrm{n} / \mathrm{cm}^{-1}: 3102$ (Ar-H), 2990(C-H), $1710(\mathrm{C}=\mathrm{O}), 1630(\mathrm{C}-\mathrm{N})$ $1590(\mathrm{C}=\mathrm{C}), 721(\mathrm{C}-\mathrm{Cl}) .{ }^{1} \mathrm{H}$ NMR $\left(\mathrm{d}, \mathrm{CDCl}_{3}\right)$ 6.4-7.9 (m, 11H, Ar-H).

\section{Evaluation of Hepatoprotective activity}

Hepatoprotective studies for compounds $\mathbf{4 , 5}$ and $\mathbf{6}$ were carried out on healthy wistar rats (190-210 g). They were divided into six groups of four animals each. The study protocol was approved by the institutional animal ethics committee for the purpose of control and supervision on animals (CPCSEA), New Delhi, India. Registration Approval No:509/01/C/CPCSEA/ dated $10^{\text {th }}$ July 2002. Carbon tetrachloride mixed with liquid paraffin (1:1) was used as hepatotoxic agent. The compounds $\mathbf{4 , 5}$ and $\mathbf{6}$ were administered in the form of aqueous suspension in $1 \% \mathrm{v} / \mathrm{v}$ of carboxymethyl cellulose for seven day after carbon tetrachloride administration. On the seventh day of the start of respective treatment the rats were anesthetized by light ether anesthesia and the blood was withdrawn from retro orbital plexus. It was allowed to coagulate for $30 \mathrm{~min}$ and serum was separated by centrifugation at $2500 \mathrm{rpm}$. The serum SGPT, SGOT, ALP and total protein were estimated in semi-autoanalyser using specific enzymatic kits (Nicolas Primal diagnostic Division, Mumbai, India). The standard drug silymarin procured from Sigma-Aldrich, USA was used as a positive control, since it protects $\mathrm{CCl}_{4}$-induced hepatotoxicity [25-26].

\section{Treatment Schedule}

Group I (Normal control): These group of rats were not given neither carbon tetrachloride nor the synthesized compounds.

Group II (CCl-treated rats): These rats were given carbon tetrachloride (1 $\mathrm{ml} / \mathrm{kg}$ ) for the first day of study to produce toxicity in the liver.

Group III (Silymarin treated rats): These rats were given a single dose of carbon tetrachloride $(1 \mathrm{ml} / \mathrm{kg})$ on first day and then silymarin $(10 \mathrm{mg} / \mathrm{kg})$ was given for 6 days

Group IV (Compound 4): These rats received were given a single dose of carbon tetrachloride $(1 \mathrm{ml} / \mathrm{kg})$ on first day and then compound $4(250 \mathrm{mg} / \mathrm{kg})$ was given for six days.

Group V (Compound 5): These rats were given a single dose of carbon tetrachloride $(1 \mathrm{ml} / \mathrm{kg})$ on first day and then compound $5(250 \mathrm{mg} / \mathrm{kg})$ was given for six days.

Group VI (Compound 6): These rats were given a single dose of carbon tetrachloride $(1 \mathrm{ml} / \mathrm{kg})$ on first day and then compound $6(250 \mathrm{mg} / \mathrm{kg})$ was given for six days.

\section{Histopathological Studies}

One animal from the treated groups showing maximal activity as thick sections and stained, using haematoxylin-eosin dye, and finally observed under indicated by improved biochemical parameters from each test, positive control, hepatotoxin and control groups were utilized for this purpose. The animals were sacrificed and the abdomen was cut open to remove the liver. Then 5 $\mathrm{mm}$ thick piece of the liver were fixed in Bouin's solution (mixture of $75 \mathrm{ml}$ of saturated picric acid, $25 \mathrm{ml}$ of $40 \%$ formaldehyde and $5 \mathrm{ml}$ of glacial acetic acid) for $12 \mathrm{~h}$ and then embedded in paraffin, using conventional methods and cut into $5 \mathrm{~m}$ microscope for histopathological changes in liver architecture, and their photomicrographs were taken.

Statistical Analysis

The mean values \pm SEM was calculated for each parameter. Percentage reduction against the hepatotoxins by the test sample was calculated by considering enzyme level difference between the hepatotoxin treated. For determining the significant inter-group difference, each parameter was analyzed separately, and one way analysis of variance (ANOVA) was carried out. Then the individual comparison of the group means values were done using Dunnett's ' $\mathrm{t}$ ' test procedure [29]. $\mathrm{P}$ values $<0.05$ were considered significant.

\section{RESULTS}

Benztriazole 1 on treatment with substituted secondary aromatic amines in presence of formaldehyde gave Mannich bases in good yields. The experimental animals after treatment with $\mathrm{CCl}_{4}$ the SGOT, SGPT and ALP levels have been increased significantly (Table 2). Significant reduction was observed for SGOT and SGPT levels in silymarin and compound $\mathbf{4}$ treated groups when compared with $\mathrm{CCl}_{4}$-treated rats. The histological studies have shown a recovery of the hepatocytes after the administration of compound 4 (N-Morpholinyl methylbenztriazole) which clearly indicated the partial protection of liver cells compared to silymarin. The liver section treated with compound $\mathbf{4}$ showed partial disappearance of fatty deposit and necrosis comparable to standard drug silymarin (Fig 1- 4). Compounds 5 and $\mathbf{6}$ showed lesser hepatoprotective activity than compound $\mathbf{4}$ and failed to show the statistical significance when compared to $\mathrm{CCl}_{4}$ treated groups.

Table 2: Effect of synthesized compounds on $\mathrm{CCl}_{4}$ induced hepatotoxicity.

\begin{tabular}{|c|c|c|c|c|}
\hline Groups & $\begin{array}{c}\text { SGOT U/ } \\
\text { mL }\end{array}$ & $\begin{array}{c}\text { SGPT U/ } \\
\text { mL }\end{array}$ & $\begin{array}{c}\text { ALP U/ } \\
\text { mL }\end{array}$ & $\begin{array}{l}\text { Total Protein } \\
(\mathrm{mg} / \mathrm{mL})\end{array}$ \\
\hline Control & $\begin{array}{c}17.0 \pm \\
2.56\end{array}$ & $\begin{array}{c}23.0 \pm \\
1.86\end{array}$ & $6.0 \pm 1.12$ & $12.29 \pm 1.10$ \\
\hline $\mathrm{CCl}_{4}$ treated & $\begin{array}{c}55.0 \pm \\
2.78^{\mathrm{a}}\end{array}$ & $\begin{array}{c}57.0 \pm \\
1.67^{\mathrm{a}}\end{array}$ & $\begin{array}{l}11.0 \pm \\
1.98^{\mathrm{b}}\end{array}$ & $13.50 \pm 1.08^{\mathrm{ns}}$ \\
\hline $\begin{array}{c}\mathrm{CCl}_{4}+ \\
\text { Silymarin } \\
\end{array}$ & $\begin{array}{l}33.0 \pm \\
2.76^{*}\end{array}$ & $\begin{array}{l}36.0 \pm \\
1.45^{*}\end{array}$ & $\begin{array}{l}9.10 \pm \\
1.32^{\text {ns }} \\
\end{array}$ & $12.85 \pm 1.12^{\mathrm{ns}}$ \\
\hline $\begin{array}{c}\mathrm{CCl}_{4}+ \\
\text { Compound } 4 \\
\end{array}$ & $\begin{array}{l}37.0 \pm \\
2.11^{* *}\end{array}$ & $\begin{array}{l}40.0 \pm \\
2.56^{* *}\end{array}$ & $\begin{array}{c}10.10 \pm \\
1.29^{\mathrm{ns}}\end{array}$ & $13.10 \pm 1.18^{\mathrm{ns}}$ \\
\hline $\begin{array}{c}\mathrm{CCl}_{4}+ \\
\text { Compound } 5\end{array}$ & $\begin{array}{l}41.0 \pm \\
1.42^{\mathrm{ns}}\end{array}$ & $\begin{array}{l}43.0 \pm \\
2.32^{\mathrm{ns}}\end{array}$ & $\begin{array}{c}10.25 \pm \\
1.45^{\text {ns }}\end{array}$ & $13.40 \pm 1.21^{\mathrm{ns}}$ \\
\hline $\begin{array}{c}\mathrm{CCl}_{4}+ \\
\text { Compound } 6\end{array}$ & $\begin{array}{l}44.0 \pm \\
1.73^{\text {ns }}\end{array}$ & $\begin{array}{c}45.0 \pm \\
1.78^{\text {ns }}\end{array}$ & $\begin{array}{c}10.30 \pm \\
1.89^{\text {ns }}\end{array}$ & $13.45 \pm 1.06^{\mathrm{ns}}$ \\
\hline
\end{tabular}

All data were expressed as mean $\pm \mathrm{SD}(\mathrm{n}=6)$

a $P<0.001$ indicates significance when compared to control.

${ }^{\mathrm{b}} P<0.01$ indicates significance when compared to control.

$* P<0.001$ indicates significance when compared to $\mathrm{CCl}_{4}$ treated

$* * P<0.05$ indicates significance when compared to $\mathrm{CCl}_{4}$ treated

ns - Not significant when compared to $\mathrm{CCl}_{4}$ treated 


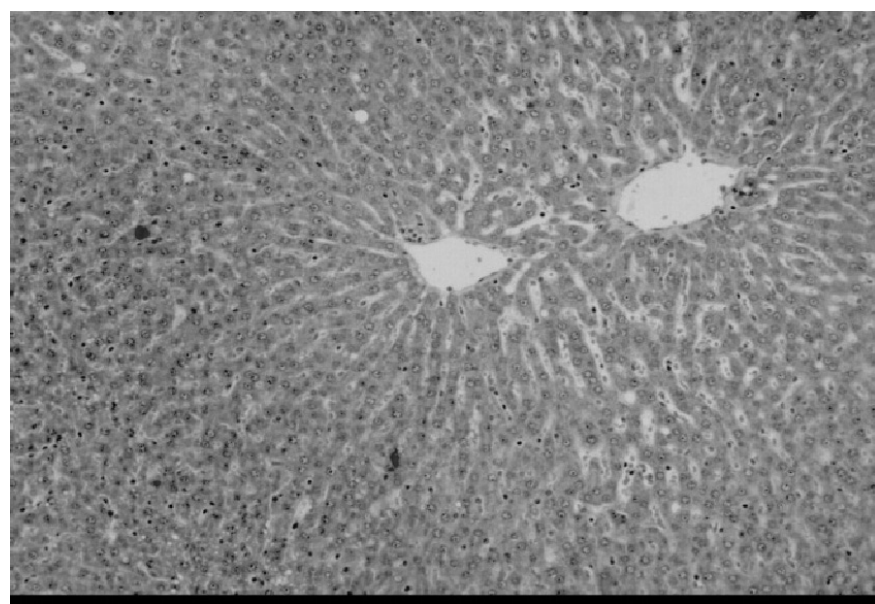

Figure 1:Normal hepatocytes without necrosis.

The liver section showing necrotic normal hepatocytes without any Necrosis abundant fatty depositions.

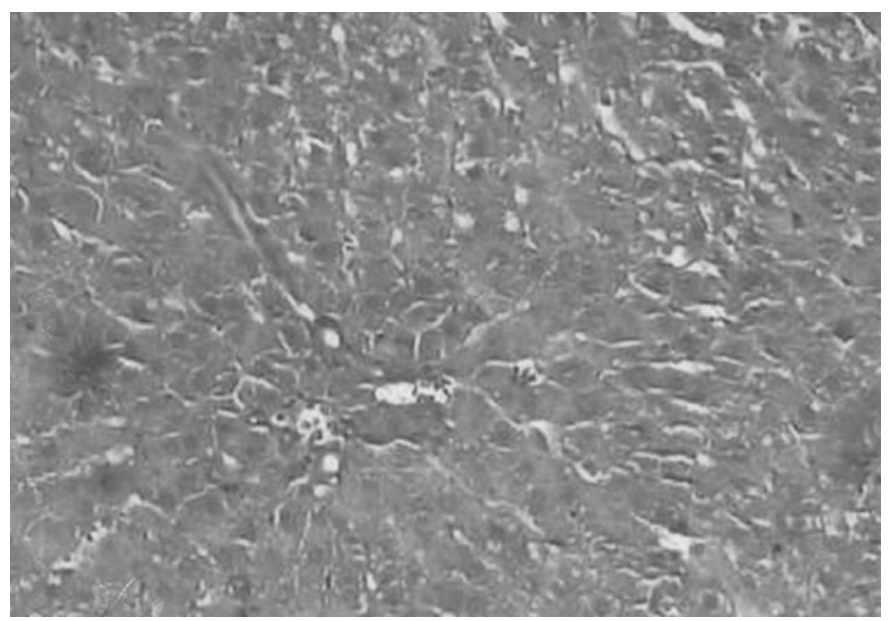

Figure 3: Liver treated with silymarin.

Sections showing good recovery with complete disappearance of fatty necrosis and fatty deposition

\section{DISCUSSION}

Mannich bases $\mathbf{2} \mathbf{- 1 0}$ obtained by synthesis were in correlation with the IR and NMR study. Since Mannich bases are reported for hepatoprotective activity, the titled compounds were screened for hepatoprotective activity. Carbon tetrachloride is a hepatotoxin commonly used for the production of experimental liver toxicity [1]. The serum transaminase level is most widely used as a measure of hepatic injury, due to its ease of measurement and high degree of sensitivity [2]. It is useful for the detection of early damage of hepatic tissue and requires less effort than that required for a histologic analysis, moreover without sacrifice of the animals. In the present study, AST levels in normal control group were in conformity with the findings of Kapoor et al [30]. Seven days pretreatment with the compounds $4(250 \mathrm{mg} / \mathrm{kg})$ protected the animals significantly from carbon tetrachloride induced hepatotoxicity. Hepatoprotective activity of compound $\mathbf{4}$ could probably due to the presence of morpholine ring since morpholine derivatives are reported to possess hepatoprotective $[27,28]$ and antioxidant activities [31].

\section{CONCLUSION}

Compound $4 \mathrm{~N}$-morpholinyl methyl benztriazole showed promising hepatoprotective activity in comparison with silymarin. The hepatoprotective

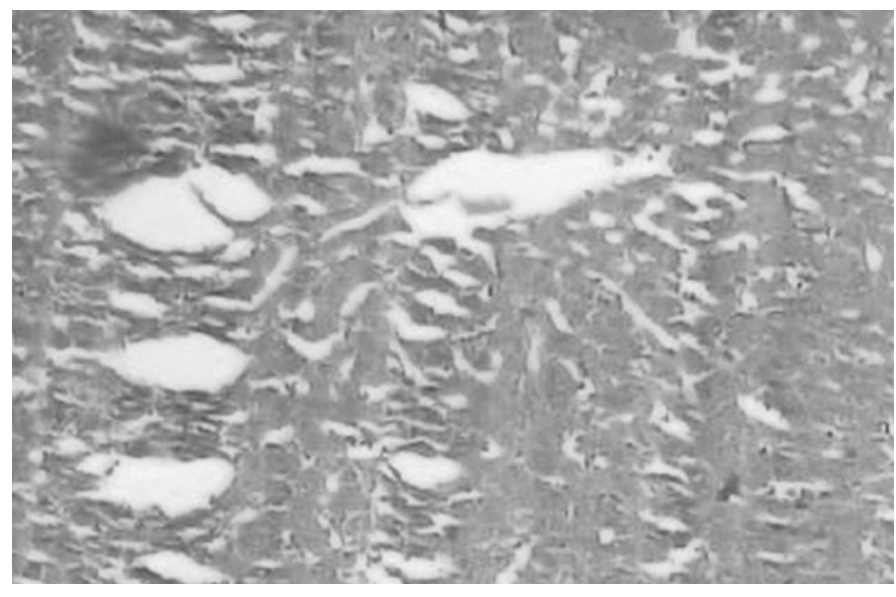

Figure 2: Liver treated with Carbon tetrachloride.

The liver section showing the development with and degeneration of liver or fatty deposition

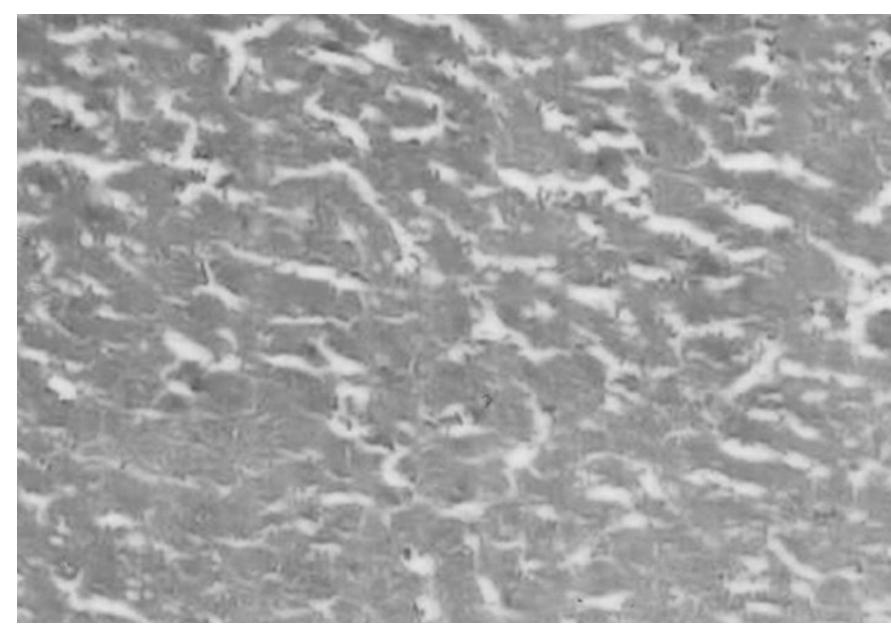

Figure 4: Liver treated with compound 4.

Section showing the partial recovery from necrosis and fatty deposition

activity of this compound $\mathbf{4}$ bearing the morpholine moiety may be due to its antioxidant activity.

\section{REFERENCES}

1. C. Girish, BC. Koner, S. Jayanthi, K.R. Rao, B. Rajesh, S.C. Pradhan. Indian J Exp Biol. 47, 257, (2009).

2. G.S. Achliya, N.R. Kotagale, S.G. Wadodkar, A.K. Dorle. Indian J Pharmacol 35, 308, (2003).

3. S.C. Pradhan, C. Girish. Indian J Med Res 124, 491, (2006)

4. M. Dheeraj, J. Sheela, V.K. Padmakar, K. Navita, Bioorg Med Chem Lett. $15,405,(2005)$.

4. J. Sheela, K. Navita, K. Deepak, S. Rakesh. Bioorg Med Chem Lett. 15, 221, (2005).

6. J. Sheela, D.M. Anju, T. Prapti. Bioorg Med Chem Lett. 17, 645, (2007).

7. A.A. Mohamed, S. Mohammad. Bioorg Med Chem Lett. 17, 3314, (2007).

8. D. Sriram, P. Yogeeswari, S.P. Reddy. Bioorg Med Chem Lett. 16, 2113 , (2006).

9. D. Sriram, P. Yogeeswari, K. Madhu. Bioorg Med Chem Lett. 15, 4502, (2005).

10. A. Chipeleme, J. Gut, P.J. Rosenthal, K. Chibale. Bioorg. Med. Chem. 15, 273, (2007). 
11. Y. Li, ZS. Yang, H. Zhang, B.J. Cao, F.D. Wang, Y. Zhang, Y.L. Shi, J.D. Yang, B.A. Wu Bioorg. Med. Chem. 11, 4363, (2005). 12. S.N. Pandeya, D. Sriram, G. Nath, E.D. Clercq. Eur. J. Med. Chem. $35,249,(2000)$

13. W. Malinka, P. Świątek, B. Filipek, J. Sapa, A. Jezierska, A. Koll. II Farmaco 60, 961, (2005).

14. M. Gokce, G. Bakir, M.F. Sahin, E. Kupeli, E. Yesilada. Arzneimittelforsch 155, 318, (2005)

15. B.S. Holla, K.N. Poojary, B. Sooryanarayana Rao, M.K. Shivananda. Eur. J. Med. Chem 37, 511, (2002).

16. M. Alkan, H. Yüksek, O. Gürsoy-Kol, M. Calapoğlu, Molecules. 13, 107, (2008).

17. B.S. Holla, B. Sooryanarayana Rao, B.K. Sarojini, P.M. Akberali, N. Suchetha Kumari. Eur. J. Med. Chem. 41, 657, (2006).

18. M. Simitsopoulou, E. Roilides, A. Maloukou, C. Gil-Lamaignere, T.J. Walsh. Mycoses. 51, 147, (2008).

19. M. Amir, K. Shikha. Eur. J. Med. Chem 39, 535, (2004).

20. A. Boido., I. Vazzana, F.Sparatore, M.L. Cenicola ML, Donnoli D, E. Marmo. Farmaco. 46, 775, (1991).

21. Y. Hirokawa, H. Yamazaki, N. Yoshida, S. Kato. Bioorg Med Chem Lett. $8,1973,(1998)$
22. B.S. Holla, B. Veerendra, M.K. ShivanandaI, B. Poojary. Eur. J. Med. Chem 38, 759, (2003)

23. M.S. Karthikeyan, D. Jagadeesh Prasad, B. Poojary, K. Subrahmanya Bhat, B.S. Holla, N. Suchetha Kumari, Bioorg Med. Chem 14, 7482, (2006).

24. V.J. Ram, M. Nath, B. Saraswat, G.K. Patnaik. Bioorg Med Chem Lett 5, 1537, (1995).

25. P. Letteron, G. Labbe, C. Degott, A. Berson, B. Fromenty, M. Larrey, D. Pessayre. Biochem Pharmacol. 39, 2027, (1990).

26. J.H. Tsai, J.Y. Liu, T.T. Wu, P.C. Ho, C.Y. Huang, J.C. Shyu, Y.S. Hsieh, C.C Tsai, Y.C. Liu. J Viral Hepat 15, 508 (2008).

27. B. Sanford. Science and practice of pharmacy. Easton: Mack Publishing Company, London, 1995.

28. V. Kapoor, K.K. Pillai, S.Z. Hussain, D.K. Balani . Indian J Pharmacol. 26, 35, (1994).

29. G. Danswan, P.J. Ramm, S. Matharu, J.B. Taylor. Arzneim-Forsch Drug Res 26, 2190, (1976).

30. Lupp A, Karge E, Wange J, Eisentraut C, Zwenizner T, Oelschläger H, Fleck C. Arzneimittelforschung. 56, 1, (2006).

31. M.C. Chrysselis, E. A. Rekka, N. Panos. J. Med. Chem. 43, 609, (2000). 